\title{
Proyectos de Neuropsicología en las aulas: experiencias e investigaciones
}

Pilar Martín Lobo. Universidad Internacional de la Rioja

Recepción: 10 de Junio de 2017 | Aceptado: 16 de Junio de 2017

Correspondencia: Pilar Martín Lobo | Correo-e: pmartinlobo@unir.net

\section{iD $0000-0002-5496-6172$}

Citar: Martín, P. (2017). Proyectos de Neuropsicología en las aulas: experiencias e investigaciones. ReiDoCrea, 6(2), 31-39.

\begin{abstract}
Resumen: La neuropsicología aplicada a la educación muestra nuevos procedimientos y metodologías innovadoras para mejorar los procesos de aprendizaje, la vida afectiva-emocional y la conducta de los alumnos en edad escolar. Luria $(1973,1983)$, explica el la estructura y el funcionamiento cerebral mediante tres bloques que sirven de fundamentación y modelo en los procesos de atención y motivación en el bloque uno; en los procesos de procesamiento de la información en las áreas sensoriales y asociativas en el bloque dos y en los procesos superiores de pensamiento y ejecutivas en el bloque tres. Además, la investigación cerebral actual, refleja la conectividad cerebral para los procesos cognitivos (Glesser et al, 2016) y sirve de orientación para identificar las causas de las dificultades de aprendizaje y los procedimientos que programas que se pueden aplicar. En este sentido, se muestran experiencias e investigaciones de la aplicación de la neuropsicología en el ámbito educativo (Martin-Lobo, 2016) que proporcionan nuevas líneas de investigación, innovación y de desarrollo de instrumentos y programas de neuropsicología educativa.
\end{abstract}

Palabras clave: Cerebro | Educación

Neuropsychology projects in the classroom: experiences and research

Abstract: Neuropsychology applied to education has revealed new procedures and innovative methodologies for improving learning processes, affective-emotional life, and the behavior of students of school age. Luria $(1973,1986)$ explains brain structure and functioning through three functional blocks. Block one serves as a foundation and model in the processes of attention and motivation; Block two is involved in the processes of information processing in the sensory and associative areas whilst block three is concerned with the higher thinking and executive processes. In addition, current brain research has demonstrated the connectivity involved in cognitive processes (Glasser et al, 2016) and serves as a guideline to identify the causes of learning difficulties and the procedures that programs can apply. In this regard, experiences and investigations of the application of neuropsychology in the educational field (Martin-Lobo, 2016) are demonstrated, providing new lines of research, innovation, and the development of instruments and educational neuropsychology programs.

Keywords: Brain | Education

\section{Introducción}

El Premio Nobel en 1906 en Medicina, Don Santiago Ramón y Cajal decía: "Todo ser humano, si se lo propone, puede ser escultor de su propio cerebro". Esta frase adquiere un sentido muy actual por los avances científicos existentes sobre el cerebro y aportan informaciones relevantes para conocer la estructura y la funcionalidad del cerebro relacionadas con el aprendizaje (Greake, 2009; Mrazik y Dombrowski, 2009). Estos conocimientos facilitan la aplicación de la neurociencia en las aulas en algunos aspectos relacionados con el aprendizaje en las aulas:

- El cerebro se transforma con el uso. Las técnicas de magnetografía cerebral muestran el funcionamiento del cerebro mientras se lee, se escribe, se soluciona un problema y en el aprendizaje en general. 
- Las experiencias moldean el cerebro y, en consecuencia, son claves los ambientes enriquecidos en la familia y en el centro educativo para el desarrollo del niño, desde su misma base neuropsicológica.

- Las investigaciones actuales ponen la atención en la creación y mantenimiento de circuitos cerebrales, no solo en la activación y localización de áreas y ayudan en la identificación de las causas de las dificultades de aprendizaje y en su tratamiento.

\section{Modelo Neuropsicológico de Luria}

Con el fin de enmarcar este conocimiento desde una perspectiva científica, con una estructura y unidad organizada y coordinada, se parte del modelo neuropsicológico de Luria $(1973,1983)$, referente para todos los investigadores, académicos, psicólogos y neuropsicólogos, puesto que profundiza en las relaciones del cerebro con la conducta y de los procesos psicológicos con los sistemas cerebrales. Este modelo tiene un enfoque multidisciplinar e interaccionista, y aporta conocimientos básicos y necesarios sobre las funciones cerebrales y la naturaleza de los procesos cognitivos desde esta perspectiva (Manga y Ramos, 2001).

Luria propone tres bloques funcionales del cerebro para explicar las funciones de los diferentes sistemas que participan en los procesos cognitivos y se caracteriza por una cooperación entre las partes que lo integran e que interactúan entre sí. Estos tres bloques funcionales corresponden en al bloque uno de alerta-motivación, atención; en el bloque dos a la recepción, procesamiento y almacenamiento de la información y en el bloque tres a la programación, control y realización de la actividad y en las funciones ejecutivas.

\section{Bloque funcional 1: alerta-motivación, atención}

Es el primer bloque de activación óptima de la corteza cerebral, necesaria para la atención y el desarrollo madurativo, cuya estructura más importante es la formación reticular ascendente y descendente y con conexiones con el córtex frontal. La atención y la concentración facilitan el proceso de aprendizaje y favorecen la activación de los bloques funcionales siguientes para aprender con efectividad. Desde edades tempranas se da una maduración y mielinización de los lóbulos frontales que facilita la activación y los procesos de inhibición.

Implicación educativa:

- Proponer la atención focalizada muy bien estructurada, organizada, con fundamentación científica, psicológica y pedagógica. Atención por parte de la dirección del colegio, del departamento de Orientación, de los profesores, tutores y con los padres en la aplicación de planes y programas para los alumnos

- Disponer de tiempos flexibles en la actividad del aula para mantener la atención y el trabajo en un proyecto o actividad iniciados.

- Diferenciar entre falta de atención y rasgos de inquietud que pueda haber en alumnos por aburrimiento en el aula; es necesario distinguir e identificar a los niños con déficits de atención e hiperactividad y evitar confusiones. A veces, se les diagnostica erróneamente de déficit de atención e hiperactividad (Lawler, 2000; Webb, 2001) y algunos de estos alumnos, incluso, pueden tener altas capacidades y se retrasa el diagnóstico de éstas por las confusiones con la atención (Moon, Zentall, Grskovic, Hall y Starmont y Spurgin, 2001, Moon, 2002). 
- Identificar alumnos que presentan dificultades escolares, teniendo en cuenta que la atención requiere habilidades neuropsicológicas que, en algunos casos, no se han desarrollado suficientemente y esto incide en el aprendizaje y en el comportamiento (Bluestones, 2000; Martín-Lobo, 2010).

\section{Bloque funcional 2: Entrada de la información por los sentidos, elaboración y almacenamiento en el cerebro}

El segundo bloque del input, corresponde a la entrada de la información por los sentidos, la elaboración y el almacenamiento de la información en el cerebro. Participan regiones del lóbulo occipital, temporal y parietal para realizar los procesos visuales, auditivos y táctiles.

De ahí la importancia de aplicar metodologías para el aprendizaje de nuevos conocimientos que se apoyen en la sensorialidad visual, auditiva, táctil y manipulativa. Los alumnos realizan los procesos cognitivos con mayor o menor rapidez, más alto nivel de comprensión y de profundidad; además, tienen mayor facilidad para acometer las tareas intelectuales más complejas. Estos procesos se realizan porque procesan la información, con conectividad entre las neuronas, comunicación entre las áreas cerebrales y conectividad interhemisférica, lo cual favorece la captación unitaria de la información y la comprensión; por ejemplo, la lateralidad homogénea del mismo lado facilita el aprendizaje y la comprensión.

Para el aprendizaje y el procesamiento de la información en el cerebro se llevan a cabo funciones que facilitan la adquisición de habilidades neuropsicológicas en los diferentes lóbulos cerebrales:

-En el lóbulo occipital se realizan funciones que favorecen habilidades visuales de motricidad, acomodación, convergencia, estereopsis, percepción visual, lenguaje visual. Las experiencias de aplicación de programas de habilidades visuales y perceptivas en las aulas de niños de cinco a diez años muestran que un porcentaje de alumnos mejoran su velocidad y comprensión lectora y cometen menos errores al leer.

-En el lóbulo temporal, las funciones auditivas favorecen la comprensión auditiva y los procesos de lenguaje. Estudios realizados sobre la aplicación de pruebas auditivas y audiometrías muestran que los alumnos que tienen buen desarrollo e integración auditiva, comprenden mejor y su rendimiento escolar es más alto que los alumnos que no adquieren habilidades de discriminación auditiva y otras relacionadas con este campo como son el ritmo, la prosodia o relación del ritmo con el lenguaje y la expresión de las palabras.

-En el lóbulo parietal, las funciones táctiles y manipulativa propician la creación de imágenes y representaciones mentales que son necesarias para la compresión de los aprendizajes más complejos, en aprendizajes matemáticos y en los procesos de creatividad.

Implicación educativa: proponer actividades y proyectos en los que se utilicen habilidades y herramientas visuales, auditivas, manipulativas, de memoria y creativas, bien fundamentadas, siguiendo los pasos del proceso creativo, coordinadas por parte de la dirección del colegio, del departamento de Orientación, de los profesores, tutores y con los padres. 


\section{Bloque funcional 3: Programación, control de la actividad y funciones ejecutivas}

El tercer bloque funcional de programación y control de la actividad se sitúa en las regiones frontales y necesita la maduración de las áreas de los bloques anteriores para su buen funcionamiento. Luria (1983) considera que estas regiones cerebrales tienen amplias conexiones con diferentes sectores de la corteza y con las estructuras subcorticales. Los lóbulos frontales están relacionados con la inhibición a estímulos irrelevantes, la conducta al fin propuesto, la direccionalidad y selectividad de procesos, la estabilidad de la actividad voluntaria, la capacidad para concentrarse en las instrucciones, para el control y para la regulación interna del lenguaje y las funciones ejecutivas (Chevalier, 2010).

En el lóbulo frontal, se realizan las funciones de pensamiento superior y las funciones ejecutivas de inhibición, planificación, flexibilidad de pensamiento y memoria de trabajo, que son claves para desarrollar todo el potencial. En la actualidad, se están aplicando programas de este tipo desde la educación infantil, como los que ha puesto en marcha el Centro ZNL, citado anteriormente.

En síntesis, el Tercer bloque realiza acciones de programación y control de la actividad, $y$, en consecuencia, de control ejecutivo y funciones ejecutivas.

Implicaciones educativas

Realizar programas de desarrollo de habilidades superiores, de Funciones Ejecutivas y asegurar las habilidades neuropsicológicas básicas que estos procesos reuieren y en las que se apoyan. Por ejemplo, un niño que tiene que resolver un problema, utiliza su atención, la visión, la audición, procesos de comprensión mediante las representaciones mentales, la planificación para saber los pasos que tiene que dar en el problema, la memoria, el pensamiento flexible y otras funciones ejecutivas. Podemos prevenir dificultades, desarrollar capacidades, resolver dificultades de aprendizaje y mejorar trastornos y dar respuesta educativa a alumnos con altas capacidades.

Las técnicas modernas de neuroimagen funcional añaden conocimientos al modelo de los bloques funcionales de Luria y permiten analizar la activación simultánea de diversas áreas cerebrales y la conectividad de las áreas entre sí para la realización de operaciones cognitivas superiores (Rosselli, Ardila y Bernal, 2015). En la utilización de imágenes de resonancia magnética multimodales del Proyecto Humano Conectoma $(\mathrm{PCH})$ y un enfoque neuroanatomical semiautomático objetivo, se delinean 180 zonas por hemisferio delimitada por los cambios bruscos en la arquitectura cortical, la función, la conectividad y / o la topografía. La parcelación y clasificador de libre disposición servirá para mejorar sustancialmente la precisión de los estudios neuroanatómicos de la organización estructural y funcional de la corteza cerebral humana y su variación entre individuos y en el desarrollo, el envejecimiento y la enfermedad (Glasser et al, 2016). En consecuencia, se pueden identificar circuitos neuronales relacionados con los diferentes aprendizajes, las emociones y la conducta.

\section{Experiencias de aplicación de la neuropsicología en la educación}

La aplicación de programas neuropsicológicos en educación infantil, para alumnos de 0 a 3 años incluye el desarrollo sensorial, motriz, (Ferré y Ferré, 2005). La aplicación de Tablas de Neurodesarrollo infantil para niños de 0 a 3 años, se realiza en varias fases que incluye la formación de los educadores infantiles; la aplicación de pautas de observación en dimensiones de visión, audición, tacto, motricidad, adaptación y 
desarrollo afectivo y emocional, junto a la adquisición de hábitos de sueño, alimentación e higiene y, en la aplicación de sus correspondientes actividades para favorecer el neurodesarrollo infantil.

En las aulas de los niños de 3 a 6 años, se aplican programas para favorecer el desarrollo sensorial, la motricidad y la coordinación visomotora relacionada con la lectura y la escritura, el desarrollo emocional y verbal (Ferré y Ferré, 2013). Además, el desarrollo auditivo, relacionado con el lenguaje y el aprendizaje de idiomas facilita el aprendizaje y el desarrollo cognitivo para preparación para la etapa educativa siguiente (Martin-Lobo, 2016).

Se aplican diferentes programas para la adquisición de las habilidades neuropsicológicas para el aprendizaje de la lectura y la escritura y el aprendizaje matemático, de lenguaje y de idiomas:

-Programa de habilidades visuales y perceptivas: para el desarrollo neurofuncional y la percepción visual que preparan para la velocidad y comprensión lectora eficiente.

-Programa de desarrollo auditivo: habilidades de la audición y la percepción auditiva para la escucha, la comprensión y expresión del lenguaje y aprender idiomas.

-Programas neuromotores: ejercicios motrices de movimientos básicos relacionados con la coordinación y la convergencia visual, el equilibrio, el tono muscular y el control postural.

-Programa de lenguaje y memoria: vocabulario, comprensión lingüística, expresión y memoria a corto y a largo plazo, nominal y procedimental.

Los resultados de los programas aplicados muestran la mejora en los procesos de aprendizaje y señalan la importancia de su aplicación para la prevención y el desarrollo desde la educación infantil. En algunos centros educativos se aplican instrumentos de valoración de habilidades visuales, auditivas, motrices, de lenguaje y memoria a los alumnos que muestran algunas dificultades para aprender.

De 7 a 11 años, se dan procesos madurativos importantes para el aprendizaje como es el caso de la atención, el razonamiento y otras funciones cognitivas (Etchepareborda, 2000).

-Programas de atención: claves para la atención focalizada, sostenida y la concentración en las tareas escolares.

-Programa de habilidades visuales y perceptivas: habilidades visuales de movimientos oculares, acomodación visual, convergencia y habilidades perceptivas se siguen desarrollando hasta los 11 años (García-Castellón, 2016).

-Programa de desarrollo de la lateralidad: lateralidad visual, auditiva y motriz que facilita la adquisición y desarrollo del sentido espacial, relacionado con los aprendizajes matemáticos y el aprendizaje (Bernabeu, 2016).

-Programas de audición, conciencia fonológica, neuromotores y de coordinación visomotora, lenguaje y memoria, mencionadas anteriormente, que se van adaptando a las demandas escolares. 
En algunos centros educativos se aplican instrumentos de valoración de habilidades visuales, auditivas, motrices, de lenguaje y memoria a los alumnos que muestran algunas dificultades para aprender e, incluso, se aplican pruebas visuales y auditivas a todos los alumnos de 6 y de 10 años para asegurar dichas habilidades para un aprendizaje eficiente.

En la edad adolescente, se dan procesos de aumento de mielinización, con mayor velocidad en el procesamiento de la información; este proceso se realiza a través del axón en desarrollo que llega a estar enrollado en una hoja de la glía (Mancini y Chabrol, 2015). "Esta mayor velocidad de comunicación entre las neuronas incide de forma positiva en el aprendizaje" (OECD Informe 2007: La comprensión del cerebro, p. 416.). El lóbulo frontal adquiere mayor desarrollo y es el periodo adecuado para la aplicación de programas de Funciones Ejecutivas a mayor nivel.

En casos de dificultades de aprendizaje en niños y adolescentes de 12 a 18 años, y en dificultades de aprendizaje de etapas anteriores, se parte de un diagnóstico neuropsicológico y se aplican pruebas que pueden ayudar a identificar las causas que provocan las dificultades de aprendizaje en cada caso. A continuación, se aplican los programas correspondientes, en pequeños grupos de cinco alumnos, durante 1 hora diaria, y relacionando los contenidos de estudio de algunas áreas curriculares. Por ejemplo, en casos de dislexia, se aplican programas de visión, de motricidad, de audición, y específicos para superar la dislexia y las dificultades de lenguaje (Pradas y De la Peña, 2016). El resultado es muy positivo, obteniendo diferencias significativas en el rendimiento escolar, a favor de los alumnos que realizan los programas neuropsicológicos.

\section{El caso de NET}

Los estudios de neurociencia indican que la atención requiere concentración y habilidades neuropsicológicas que inciden en el comportamiento y en el aprendizaje (Bluestones, 2000; Martín-Lobo, 2010). Uno de estos estudios es el caso de Net; un niño con 9 años y dificultades de atención y de concentración en clase que se movía continuamente en su silla y suspendía; los resultados de las pruebas que le aplicaron mostraron que no tenía habilidades visuales apropiadas para el estudio, su sistema vestibular y su motricidad no habían adquirido el nivel de desarrollo suficiente porque se cayó de pequeño y estuvo escayolado sin poder moverse durante un largo periodo de tiempo que le impidió desarrollar su sistema motriz y su lateralidad suficientemente. A los dos meses de aplicarle los programas visuales, vestibulares, de motricidad y nutricional que necesitaba se observó una clara mejoría. Se comprobó posteriormente que Net tenía altas capacidades, aunque mostraba dificultades claras de atención y superó las dificultades mediante la aplicación de los programas adecuados a su necesidad de mejora atencional.

\section{Investigaciones}

Se muestran dos investigaciones reflejadas en dos artículos, producto del trabajo del Grupo de Investigación de Neuropsicología aplicada a la Educación de la UNIR.

-Megino Elvira,L, Martín-Lobo P. y Vergara-Moragues E. (2016) Influence of eye movements, auditory perception and phonemic awareness in the reading process Journal of educational research, 109, 567-573. doi: 10.1080/00220671.2014.994197. Factor de impacto JCR:1.218 Q2 
El artículo presenta un estudio sobre la relación de los movimientos oculares, la discriminación auditiva y la conciencia fonémica con el proceso lector. Los resultados tras el análisis correlacional indican que las variables estudiadas están relacionadas con la lectura y muestra que los alumnos que presentan dificultades de movimientos oculares o de discriminación auditiva obtienen un nivel significativamente inferior en lectura. Se propone un programa de intervención con base neuropsicológica para desarrollar las habilidades lectoras. Todos estos aspectos son ampliamente estudiados en el Máster de Neuropsicología y Educación.

http://www.tandfonline.com/doi/full/10.1080/00220671.2014.994197?scroll=top \&needAccess $=$ true \&

-López-Fernández, V y Llamas-Salguero, F (2017). Neuropsicología del proceso creativo. Un enfoque educativo. Revista complutense de Educación (En impresión).

El artículo aporta un mejor entendimiento de la creatividad desde un enfoque neuropsicológico, dado el carácter multidimensional del constructo. Se presenta una revisión de investigaciones que estudian la creatividad desde la neurociencia y se propone una visión de las funciones neuropsicológicas implicadas en dicho proceso, en función de la fase en la que nos encontramos ante actividades: preparación, incubación, iluminación y verificación. Los resultados muestran las funciones cognitivas de especial relevancia en cada una de las fases y estructuras cerebrales con gran activación en las distintas etapas del proceso creativo. Se proponen nuevas líneas para investigaciones desde la perspectiva neuropsicológica en el estudio de la creatividad.

\section{Áreas de Investigación}

El área de investigación del grupo se encuadra dentro del campo de la evaluación, detección, e intervención en neuropsicología aplicada a la educación en el aula, para la prevención y el desarrollo, las dificultades y trastornos del aprendizaje, con el objetivo de mejorar el rendimiento escolar y la calidad educativa en los estudiantes en edad escolar.

Actualmente las principales líneas de investigación del grupo son:

Diseño y validación de instrumentos de valoración en Neuropsicológica Educativa.

Estudio de procesos neuropsicológicos de aprendizaje y elaboración y de programas para el aula. Neurotecnología.

Neuropsicología para Dificultades y Trastornos del Aprendizaje en el ámbito educativo.

\section{Proyectos}

Validación de la Prueba Neuromotriz EVANM y Prueba de Lateralidad PVL.

En conclusión, se puede afirmar que la neuropsicología se está aplicando a los procesos de aprendizaje en las aulas de las diferentes etapas educativas y para mejorar las dificultades de aprendizaje en algunos centros educativos. Para ampliar estas experiencias, es necesario llevar a cabo programas de formación de directivos de centros educativos, de psicólogos, de orientadores y de profesores con el fin de incorporar la neuropsicología con el rigor científico y la metodología que requiere. Las limitaciones se centran en las dificultades existentes para evidenciar las mejoras 
educativas y de aprendizaje que producen este tipo de aplicaciones. Esta situación hace pensar en líneas de futuro de investigación, en desarrollo de programas, en la posibilidad de extender su aplicación y, al mismo tiempo, en realizar la investigación en el aula y en el centro educativo, mediante un trabajo en equipo de investigadores, profesores de universidad y profesores del propio centro educativo; para lo cual es necesario obtener la financiación de empresas, fundaciones y del propio Ministerio de Educación, tal y como se realiza ya en Centros Internacionales de prestigio, como el de Transferencia de la Neurociencia a la Educación, ZNL, de la Universidad de ULM, en Alemania y el Centro de Neurociencia y Educación de la University of Cambridge.

\section{Referencias}

Barrero Borrallo, Vergara-Moragues, y Martin-Lobo. (2016). Avances neuropsicológicos para el aprendizaje matemático en educación infantil: la importancia de la lateralidad y los patrones básicos del movimiento. Edma, 0-6: Educación matemática en la infancia, 4 (2), 22-31.

Bernabeu, E. (2016). Programas de desarrollo de la lateralidad, mejora del esquema corporal y organización espacio-temporal. Intervención en dificultades de aprendizaje. En Martín-Lobo (2016). Procesos y Programas de Neuropsicología Educativa. Ministerio de Educación, Cultura y Deporte: Centro Nacional de Investigación e Innovación (CNIIE).

Bluestone, J. P. (2000). Disorders: Attention Déficit Hyperactivity Disorder. Rieved from The HANDLE Instiute. Washington.

Chevalier, N. (2010). Executive functions in children: concepts and developments. Canadian Psychology - Psychology Canadienne, 51(3), 149163.

García-Castellón, M.C. (2016). Procesos y programas de habilidades visuales y perceptivas para la lectura eficaz. En Martín-Lobo (2016). Procesos y Programas de Neuropsicología Educativa. Ministerio de Educación, Cultura y Deporte: Centro Nacional de Investigación e Innovación (CNIIE).

Ferré, J. (2005). El desarrollo neuro-senso-psicomotriz de los 3 primeros años de vida. Lebón: Madrid.

Ferré, J. y Ferré, M. (2013). Neuro-psico-pedagogía infantil. Bases neurofuncionales del aprendizaje cognitivo y emocional. Barcelona: Lebón.

Glasser, M., Coalson, Emma C. Robinson, Hacker, C., Harwell, J; Yacoub, E., Ugurbil, K.; Andersson, J.; Beckmann, J.; Jenkinson, M.; Smith, S. \& Van Essen. D. (2016). A multi-modal parcellation of human cerebral cortex. Nature, 536, 171-178.

López-Fernández, V y Llamas-Salguero, F (2017). Neuropsicología del proceso creativo. Un enfoque educativo. Revista complutense de Educación (En impresión).

Luria, A. R. (1973). The working brain. London: Penguin books.

Luria, A. R. (1983). Las funciones psíquicas superiores y su organización cerebral. Barcelona: Fontanella.

Mancini, J. y Chabrol, B. (2015). Desarrollo neurológico. ECM-Pediatría, 50, 1-11.

Manga, D. y Ramos, F. (2011). El legado de Luria y la neuropsicología escolar. Psychology, Society, \& Education 2011, Vol.3, № 1, pp.1-13 ISSN 2171-2085.

Martin-Lobo, P. (2010). La lectura. Procesos neuropsicológicos del aprendizaje, estudio de casos, diagnósticos y programas de intervención. Barcelona: Lebón.

Martín Lobo, P. (Coord.) (2016). Procesos y programas de neuropsicología educativa. Madrid: Ministerio de Educación, Cultura y Deporte: Centro Nacional de Investigación e Innovación (CNIIE).

Martín Lobo P. y Vergara-Moragues, E. (Coords) (2016). Procesos e instrumentos de evaluación neuropsicológica educativa. Madrid: Ministerio de Educación, Cultura y Deporte: Centro Nacional de Investigación e Innovación (CNIIE).

Megino-Elvira, L. Martín-Lobo, P. y Vergara-Moragues, E. (2016): Influence of eye movements, auditory perception, and phonemic awareness in the reading process, The Journal of Educational Research. DOI: 10.1080/00220671.2014.994197. 
OCDE (2007). Understanding the Brain: The Birth of a Learning Science. OECD (Organisation for economic co-operation and development.

Pradas, S y De la Peña, C. (2016). Programas para superar las dificultades del lenguaje. En Martín-Lobo (2016). Procesos y Programas de Neuropsicología Educativa. Ministerio de Educación, Cultura y Deporte: Centro Nacional de Investigación e Innovación (CNIIE).

Rosselli, M., Ardila, A. y Bernal, B. (2015). Modelo de conectividad de la circunvolución angular en el lenguaje: metaanálisis de neuroimágenes funcionales. Rev Neurol, 60, 495-503. 